\title{
openheart In vivo biocompatibility and pacing function study of silver ion-based antimicrobial surface technology applied to cardiac pacemakers
}

\author{
James Shawcross, ${ }^{1}$ Ameet Bakhai, ${ }^{2}$ Ali Ansaripour, ${ }^{3}$ James Armstrong, ${ }^{1}$
} David Lewis, ${ }^{1}$ Philip Agg, ${ }^{1}$ Roberta De Godoy, ${ }^{4}$ Gordon Blunn ${ }^{4}$

To cite: Shawcross J, Bakhai A, Ansaripour A, et al In vivo biocompatibility and pacing function study of silver ion-based antimicrobial surface technology applied to cardiac pacemakers. Open Heart 2017;4:e000357. doi:10.1136/ openhrt-2015-000357

Received 30 0ctober 2015 Revised 14 July 2016 Accepted 2 August 2016
CrossMark

${ }^{1}$ Accentus Medical Ltd, Didcot, UK

2Department of Cardiology, Royal Free London NHS Trust, London, UK

${ }^{3}$ Department of Cardiology, Barnet General Hospital, London, UK

${ }^{4}$ Institute of Orthopaedics and Musculo-Skeletal

Science, Division of Surgery \& Interventional Science, University College London, London, UK

\section{Correspondence to} Dr James Shawcross; james. shawcross@accentus-medical. com

\section{ABSTRACT}

Introduction Evidence suggests that the rate of cardiovascular implantable electronic device (CIED) infections is increasing more rapidly than the rates of CIED implantation and is associated with considerable mortality, morbidity and health economic impact. Antimicrobial surface treatments are being developed for CIEDs to reduce the risk of postimplantation infection within the subcutaneous implant pocket.

Methods and analysis The feasibility of processing cardiac pacemakers with the Agluna antimicrobial silver ion surface technology and in vivo biocompatibility were evaluated. Antimicrobially processed $(n=6)$ and control pacemakers $(n=6)$ were implanted into subcutaneous pockets and connected to a part of the sacrospinalis muscle using an ovine model for 12 weeks. Pacemaker function was monitored preimplantation and postimplantation.

Results Neither local infection nor systemic toxicity were detected in antimicrobial or control devices, and surrounding tissues showed no abnormal pathology or over-reactivity. Semiquantitative scores of membrane formation, cellular orientation and vascularity were applied over five regions of the pacemaker capsule and average scores compared. Results showed no significant difference between antimicrobially processed and control pacemakers. Silver analysis of whole blood at 7 days found that levels were a maximum of 10 parts per billion (ppb) for one sample, more typically $\leq 2 \mathrm{ppb}$, compared with $<<2 \mathrm{ppb}$ for preimplantation levels, well below reported toxic levels.

Conclusions There was no evidence of adverse or abnormal pathology in tissue surrounding antimicrobially processed pacemakers, or deleterious effect on basic pacing capabilities and parameters at 12 weeks. This proof of concept study provides evidence of basic biocompatibility and feasibility of applying this silver ion-based antimicrobial surface to a titanium pacemaker surface.

\section{INTRODUCTION}

An increasing number of cardiac devices are being implanted globally each year, including implantable loop recorders, permanent pacemakers, implantable cardioverter-defibrillators (ICDs) and cardiac

\section{KEY MESSAGES}

What is already known about this subject? An increasing number of cardiac devices are being implanted globally each year, which are collectively known as cardiovascular implantable electronic devices (CIEDs). Although the use of a CIED is associated with a lower risk of death and a better quality of life, the onset of a postoperative devicerelated infection can lead to devastating and costly clinical complications. Recent data suggest that the rate of CIED infection may be rising faster than the rate of CIED implantation. Approaches are therefore being investigated to reduce the risk of such infections.

What does this study add?

This study demonstrates the feasibility of processing cardiac pacemakers with the silver ion-based Agluna surface technology and in vivo biocompatibility. By treating the cardiac pacemaker as an integral part of the manufacturer's standard manufacturing processes, it avoids the use of approaches such as antibioticloaded pouches or the application of topical agents at the time of surgery.

How might this impact on clinical practice? The key aim of this approach is to reduce the risk of infection in the first 3-6 months, following surgical implantation of the CIED. This will improve patient outcomes and reduce the cost of treating infections associated with such procedures. By treating the device surface as an integral part of the manufacturer's standard manufacturing processes, it reduces the time and procedural complexity of other approaches.

resynchronisation therapy (CRT) devices (collectively termed cardiovascular implantable electronic devices(CIEDs)).

Implantation rates are rising for two reasons: an increase in the range of clinical indications for which CIEDs have been approved and also the fact that the population is ageing and therefore demand is greater. Although the use of a CIED is associated with a lower risk of death and a better quality of life, the 
onset of a postoperative device-related infection can lead to devastating and costly clinical complications. ${ }^{1}$ Historically, reported rates of these infections range from $0 \%$ to as high as $20 \%{ }^{1}$ in some series. However, recent data suggest that the rate of CIED infection may be rising faster than the rate of CIED implantation. ${ }^{1}$ As well as causing considerable morbidity and prolonged hospitalisation, mortality rates remain high and the economic burden of CIED infection management is high, both for patients and third-party payers. ${ }^{1-5}$ There are a number of studies examining the cost of combined medical and surgical treatment of CIED-related infections, indicating that cost typically varies between US $\$ 25000$ for permanent pacemakers and US\$50000 for ICD and CRTs. ${ }^{46-8}$ A US retrospective study ${ }^{8}$ of 200219 Medicare patients (2007 calendar year) reported 5817 admissions for infection as well as significant increases in mortality (admissions and long term) and length of stay depending on CIED type. The standardised, adjusted total admission cost ranged between US $\$ 28676$ for pacemakers and US\$53349 for complex devices (Cardiac Resynchronisation Therapy Defibrillator CRT-D, or a Cardiac Resynchronisation Therapy Pacemaker (CRT-P) and ICDs). Costs of intensive care, length of stay and supplies, including replacement CIED, were the highest proportion of the costs. The standardised, adjusted incremental cost was between US $\$ 14360$ and US\$16498 depending on CIED type.

The purpose of this study was to evaluate the biocompatibility of a silver ion-based antimicrobial surface technology applied to the outer can of a pacemaker and its impact on basic pacemaker function. The study would also provide an opportunity to examine for signs of any in vivo bacterial colonisation or infection associated with implantation. The study was not designed to evaluate any specific antimicrobial effect or powered to detect a reduction in infection rate.

\section{FEASIBILITY OF ANTIMICROBIAL SURFACE PROCESSING OF PACEMAKERS}

The Agluna antimicrobial surface is produced by an advanced electrochemical process, developed and owned by Accentus Medical, Oxfordshire, UK. The process relies on an anodising technique that forms a passive layer integral to the surface and is primarily applicable to medical devices manufactured from titanium and its alloys. The process creates 3-5 $\mu \mathrm{m}$ diameter reservoirs in the surface of the implant that contains amorphous hydrous titania. Silver ions $\left(\mathrm{Ag}^{+}\right)$are incorporated into the surface by ion exchange primarily into the hydrous titania. $\mathrm{The}^{\mathrm{Ag}^{+}}$ions inhibit the local colonisation of pathogens on the device surface and hence the formation of biofilm, while also eluting in a controlled manner into the local environment during the critical weeks and months of the postoperative period, reducing the risk of localised infection. A number of in vitro studies have been performed to demonstrate the safety and efficacy of the approach. The efficacy has been shown against a number of Gram-positive bacteria (eg, Staphylococcus aureus and Staphylococcus epidermidis), Gram-negative bacteria (eg, Pseudomonas aeruginosa) and fungi (eg, Candida albicans) commonly associated with medical device-related infections. This antimicrobial surface is in clinical use, applied to custom limb salvage orthopaedic implants and a CE mark-approved Modular Endoprosthetic Tumour System (METS), both distributed by Stanmore Implants Worldwide, Elstree, UK, which replace large segments of resected bone. Studies report greater than $50 \%$ reduction in infection in this high-risk patient cohort as well as a number of additional observed clinical benefits. ${ }^{910}$

Initial feasibility of antimicrobial surface processing of cardiac devices was assessed prior to this in vivo evaluation phase, by processing a series of explanted pacemakers (after pacemaker generator change) with the antimicrobial technology and assessing the effect on pacing and residual battery function. This work showed no deleterious effect on the basic pacing function of the pacemaker, and demonstrated that this antimicrobial surface would be feasible for subsequent evaluation of in vivo biocompatibility and device function.

\section{OBJECTIVES OF IN VIVO EVALUATION}

The objective of this in vivo study was to determine whether antimicrobial surface processing of the titanium surface of cardiac pacemakers would alter local, soft tissue biocompatibility compared with control implants. The study would also allow assessment of biofilm incidence, seen as precursor to implantable device infection. In addition, this study would confirm whether pacemaker function could be maintained in vivo, prior to any possible clinical evaluation. To enable an accurate comparison, antimicrobial surface processed and non-processed control pacemakers were implanted in the same control subjects. The antimicrobial surface was not applied to the pacing leads primarily as the process involves an anodising technique that is only applicable to medical devices consisting of titanium, tantalum or zirconia (and their alloys). There are studies that report metal-catalysed degradation of polyurethane. ${ }^{11}$ The conductor is often a nickel/cobalt/chromium/molybdenum alloy with elements added to improve conductivity such as a silver core. ${ }^{12}$ The degradation is most likely due to the catalytic reduction of hydrogen peroxide to water at the silver metal surface, which then by direct electrical contact with the conductor is able to increase the rate of galvanic corrosion. This leads to an increase in the concentration of transition ions locally, and accelerated hydroxyl radical production, by well-known interaction with hydrogen peroxide, resulting in further polyurethane degradation. Electrical isolation is therefore the recommended approach to eliminate unwanted galvanic corrosion. The silver used in this antimicrobial surface is in the form of adsorbed silver ions incorporated into the semiconductor oxide film, which is not in electrical contact with the conductor and so will not be able to drive 
the accelerated galvanic corrosion, leading to degradation of the polyurethane. This reduces the possibility of significant deleterious effect on the polyurethane-coated leads that may also be located in the device pocket.

\section{MATERIALS AND METHODS \\ Experimental in vivo protocol}

An ethics committee-approved ovine model was used to test the in vivo performance of these devices. In our model, antimicrobial surface processed and non-processed control pacemakers were implanted at the same procedure between muscles in the back, on either side of the spine of the same sheep to maximise the comparison potential for the animals to act as their own controls. To provide considerable confidence for future human studies and simulate natural bacterial colonisation conditions, follow-up of the animals was continued for 12 weeks ( 84 days). Although by design, this study was not Good Laboratory Practice (GLP) compliant, the implantation was performed in a GLP-approved facility at the Royal Veterinary College, Hertfordshire, UK. Therefore, the implantation procedure, the in-life phase and the retrieval of implants followed GLP guidelines and was ethics committee approved. The antimicrobial surface process was only applied to the outer pacemaker titanium casing and not to any other component, such as the device header or the leads. To ensure comparison of antimicrobial processed devices for leading manufacturers, 12 single chamber (VVI) pacemakers and corresponding electrodes were purchased from the four most common UK manufacturers, as shown in table 1 , and pacing programmers utilised for the duration of the study. This enabled us to physically and closely simulate human conditions where various pacemaker generators of differing sizes and shapes are used, with differing final pacemaker construction.

The surface of six cardiac pacemaker outer titanium casings was antimicrobially processed by Accentus Medical and then resterilised using ethylene oxide (Andersen Caledonia, Bellshill, UK) and packed ready for implantation (the manufacturers supplied control sterile pacemakers). The corresponding control pacemakers were not handled in any other way, other than externally testing pacing parameters including battery life and pacing impedance. Pacemaker function was tested preimplant while in the packaging (and for test generator models, postantimicrobial processing and

\section{Table 1 Implants used}

\begin{tabular}{llll}
\hline Supplier & Model & $\begin{array}{l}\text { Number } \\
\text { supplied }\end{array}$ & $\begin{array}{l}\text { Agluna } \\
\text { processed }\end{array}$ \\
\hline Boston Scientific & Altrua 20 SSIR-1 & 4 & 2 \\
Medtronic & Adapta ADSR01 & 4 & 2 \\
St Jude & Zephyr XL SR & 2 & 1 \\
Sorin & Reply SR & 2 & 1 \\
\hline
\end{tabular}

preimplant). Threshold testing was done manually to see and feel muscle contraction at 60 beats per minute and then at $10 \mathrm{~V}$ to confirm no other adverse reactions in VOO pacing mode, as striated muscle signals could not be detected while the animal was under anaesthesia. The same pacing and threshold tests were performed for each pacemaker. By design, there was no intention nor ethical approval to pace the heart in this study.

\section{Implantation}

The day of surgery was referred to as S0. Prior to surgery, blood samples were taken to determine baseline levels of silver, and clotted and non-clotted blood samples were stored at $-73^{\circ} \mathrm{C}$ until required. At surgery, a dorsal, longitudinal incision measuring $\sim 5 \mathrm{~cm}$ was made, positioned $\sim 3 \mathrm{~cm}$ to either side of the spine. The underlying fascia was dissected and the dorsal surfaces of sacrospinalis muscle were accessed by further blunt dissection. After confirming access to muscle, the electrode was placed on the muscle with the pacing tip at least $5 \mathrm{~cm}$ or more away from the pacemaker pocket space, and then connected to the pacemaker generator. The pacemaker was positioned on the surface of the muscle, away from the tip, with spare electrode coiled below the generator, simulating human implantation conditions other than the electrode not being placed via vessels into the heart. A non-resorbable suture was used to tie the pacemaker electrode in place, around the provided sleeves. There was surprisingly good space above the muscle and the thick fat layers to place the pacemaker comfortably without causing any non-smooth pocket profiles or risk of erosion against vertebral or thoracic bones. The wound was closed in three layers with resorbable sutures. This procedure was repeated on the other side of the spinal column. In all cases, the left-hand side received a control pacemaker first, and the right-hand side received the antimicrobially processed pacemaker, with sterile conditions maintained on both sides. Two experienced veterinarian surgeons performed the procedures with an experienced pacing cardiologist in theatre to programme, activate and test the pacing function (preprocedure, periprocedure and postprocedure), and test electrode thresholds and impedance as well as confirm muscle contraction. Each procedure took about $25 \mathrm{~min}$, with separate aseptic techniques for both procedures on each sheep under general anaesthesia. Pacemakers were also activated postanaesthesia to visualise muscle contraction, but then left inactive to avoid animal discomfort. Intermittent pacing activations were made to check pacing threshold in both bipolar mode and unipolar mode confirming required function. Antibiotics were administered in compliance with ethical approval and study protocols. This is standard practice in this study model and no effect on wound healing or end point was anticipated. Animals were then returned to individual pens. During this extensive experimental condition, animals were observed weekly for operative healing, infection symptoms and reactions externally. 


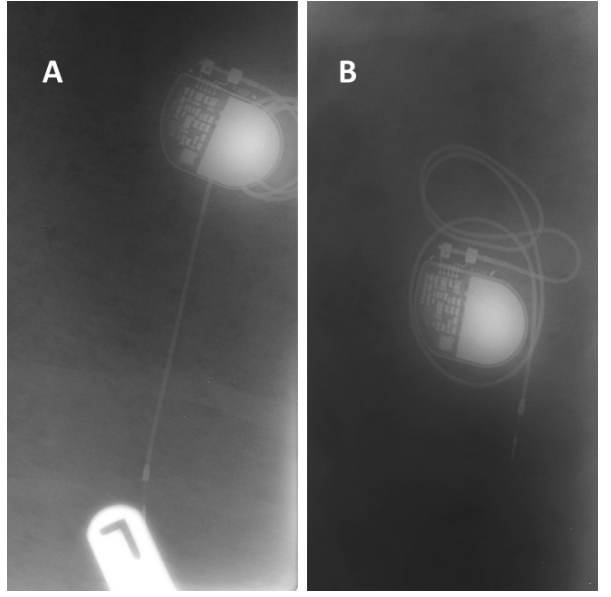

Figure 1 Radiographs of in situ pacemakers control (A) and antimicrobially processed surface (B).

Blood samples were taken on days S0, S7, S14, S28, S56 and S84 and stored in a freezer.

\section{Implant retrieval}

After sacrifice with an overdose of sodium pentobarbitone, animals were positioned in sternal recumbency, and a radiograph was taken to determine the position of the pacemaker. Using the same sterile conditions and operating conditions as during implant, the pacing pockets were entered using aseptic technique, creating a new incision just dorsal to the original incision site. Wound healing was assessed prior to incision and pacemakers checked for migration and activated to check parameters and muscle contraction. Digital photography records were undertaken throughout the surgeries, including prior to entering the pacemaker capsules and after exposing the implant pocket sites. The soft tissue surrounding the pacemaker was isolated. If a fluid-filled pocket had formed, then the fluid was aseptically aspirated and samples sent for microbiological assessment. The soft tissue surrounding the pacemaker was breached with a sterile scalpel and a swab stick inserted through the opening and manipulated over the surface of the pacemaker and the adjacent soft tissue. The swab was sent off for microbiological assessment, examining for bacterial isolates after 48 hours aerobic and anaerobic incubation. Samples of the tissue surrounding the pacemaker were taken and frozen at $-73^{\circ} \mathrm{C}$ and sent for silver measurement by inductively coupled plasma mass spectroscopy (ICP-MS), where required. Pacing generators and electrodes were stored separately in sterile zip-locked bags and photographed for biofilm formation. Other samples of surrounding tissue were taken and processed for paraffin wax embedded histology. Samples of spleen, heart, liver, kidney and lungs were fixed in formal saline and retained.

The measurement of inflammation was blinded, based on an assigned code, which was disclosed only after the analysis and performed by two independent analysts. This used a semiquantitative scoring system for membrane formation, cellular orientation and vascularity. As the data were not normally distributed, as confirmed by Kolmogorov-Smirnov test, a non-parametric Mann-Whitney $\mathrm{U}$ test comparing two independent variables was used, where $\mathrm{p}<0.05$ was considered to be significant. The number of macrophages in the capsule surrounding the implants was estimated by counting cells in a $10 \times$ objective field of view, in 10 different regions. A comparison was made with tissue surrounding control implants and analysed using energy dispersive X-ray (EDX) analysis to determine the amount of silver within the surrounding tissues. Two of the antimicrobially processed pacemakers were taken and examined under scanning electron microscopy-EDX for silver content. The retrieved pacemakers were assessed to check that they were working appropriately again after removal from the sheep, confirming battery and programming parameters only. All electrode material was also removed. Residual silver measurement on the antimicrobially processed pacemakers was then performed using acid extraction and ICP-MS.

\section{RESULTS}

Figure 1 indicates the position of the Sorin Reply SR pacemakers on radiographs confirming the implants have remained in place during the 12-week period of insertion.

The appearance of the tissue surrounding the implant and the capsule where it was located was consistent. In some cases, capsules displayed slight evidence of inflammation but were all well vascularised. All pacemakers could easily be removed, without any tissue remaining attached to its surface, leaving an imprint on the tissue surface, with little evidence of inflammation. By way of example, figure 2 shows postmortem photographs of a control Sorin Reply SR pacemaker being retrieved; clearly a fibrous tissue membrane, which is translucent, covers the pacemakers on its dorsal surface. This membrane appears to be relatively avascular with little evidence of
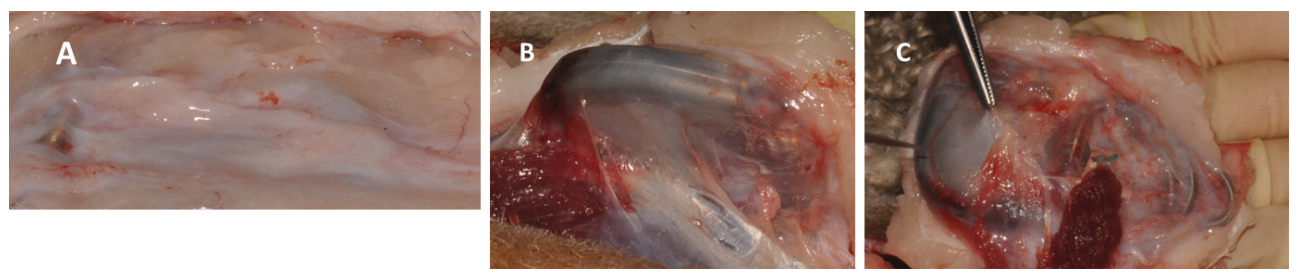

Figure 2 Retrieval photographs (A-C) from around a control Sorin Reply SR. 

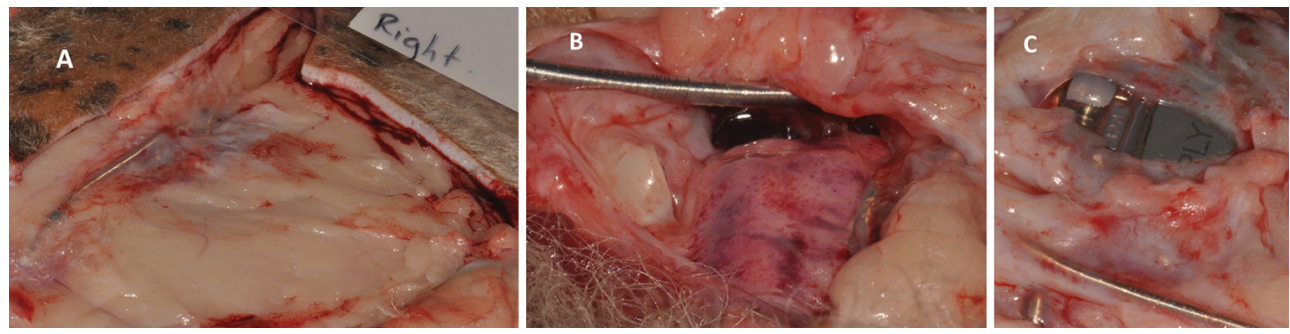

Figure 3 Retrieval photographs $(A-C)$ from around an antimicrobially processed Sorin Reply SR pacemaker.

inflammation and small volume of exudate in the pocket surrounding this implant.

The tissue surrounding the antimicrobially processed pacemaker on the right-hand side of the animal (figure 3) has a more vascular capsule (than that in figure 2), as evidenced by its redder appearance. The tissues overlying the pacemaker appear to be thicker and more inflamed. There was also clear exudate within the pocket enclosing the pacemaker on this side.

Swabs taken aseptically from the surface of the pacemaker at retrieval and then microbiologically assessed showed no evidence of any bacterial growth, indicating that all the pacemakers were located within a sterile pocket.

Histological examination of the soft tissue capsules surrounding the pacemakers using H\&E staining from around both antimicrobially processed and non-processed control pacemakers showed that the capsules surrounding the implants were composed of fibrous tissue with some macrophage infiltration, though with no evidence of any plasma cells. The fibrous tissue was well aligned and parallel to the surface of the capsules and in many instances was producing a well-aligned collagenous tissue. In other regions of the same pacemakers, and indeed in other pacemakers, irrespective of whether they were antimicrobially processed or control devices, a layer of macrophages could be observed at the surface of the tissue adjacent to the implant. The surface of the capsule adjacent to the antimicrobially processed pacemaker or untreated pacemaker is composed of macrophages, whereas deeper in the capsule, the layer is composed of more fibroblasts.

In places, a brown flaky material is seen within macrophages, often adjacent to blood vessels in the capsule surrounding both surface processed and non-processed control pacemakers. EDX confirmed that this material was composed of iron and was likely a breakdown product of haemoglobin, often referred to as haemosiderin. There was no evidence of any silver-containing particles in the capsule tissue surrounding the antimicrobially processed pacemakers, using EDX.

The quantification of the interface surrounding all the pacemakers by measuring the thickness of the capsule and a comparison made between the tissues surrounding antimicrobial processed and control pacemakers indicated the average thickness for these samples was in the range $500-700 \mu \mathrm{m}$. There was no difference in the average capsule thickness surrounding the antimicrobial processed and control pacemakers ( $\mathrm{p}=0.937$; MannWhitney U non-parametric analysis).

The cellularity, vascularity and production/organisation of collagen fibrous tissue scoring used a semiqualitative score from 1 to 5 , with 1 being low and 5 being high with five regions of the capsule for each pacemaker scored. The average of these scores from the six animals was measured for antimicrobially processed and control implants, and the mean compared using a Mann-Whitney U non-parametric analysis. For all measurements, results showed no significant difference between antimicrobially processed and control pacemakers (cellularity: $\mathrm{p}=0.81$; vascularity: $\mathrm{p}=0.132$; collagen: $\mathrm{p}=0.81$ ).

There was no significant difference in the number of macrophages in fields of view in capsules surrounding antimicrobially processed and control pacemakers. Due to the different structure of the capsules between sheep, the data show a large interquartile spread of the data for both groups with micrographs showing the variability of the tissues surrounding the pacemakers. The number of macrophages within the capsules was also variable. In tissues from around both antimicrobially processed and control pacemakers, macrophages tended to be more prominent in number towards the surface of the capsule.

Silver analysis of whole blood at 7 days found that levels were a maximum of $10 \mathrm{ppb}$ (parts per billion) for one sample, more typically $\leq 2 \mathrm{ppb}$, compared with $<<2 \mathrm{ppb}$ for preimplantation levels (see table 2, limit of detection $0.6 \mathrm{ppb}$ ). Only day 1 and day 7 blood samples were analysed, as supporting elution studies have shown that silver ion release is most rapid in the first few hours and days, followed by an exponential fall off over a period of weeks to months to below detectable limits. These levels are considerably below reported clinical and toxic levels based on cellular response. ${ }^{13}$

\begin{tabular}{lll} 
Table 2 & Silver concentration in whole blood \\
\hline & Day $\mathbf{1}(\mathbf{p p b})$ & Day $\mathbf{7}(\mathbf{p p b})$ \\
\hline Sorin & $<0.6$ & 2 \\
Medtronic & 1 & $<0.6$ \\
St Jude & 1 & 10 \\
Boston & 2 & 2 \\
\hline
\end{tabular}

ppb, parts per billion. 


\begin{tabular}{ll} 
Table 3 & Silver concentration in local tissue \\
\hline & Tissue (ppm) \\
\hline Sorin & 0.39 \\
Medtronic & 2.4 \\
St Jude & 2.7 \\
Boston & 0.2 \\
\hline
\end{tabular}

ppm, parts per million.

Local tissue silver concentrations were in a range between 0.2 and $2.7 \mathrm{ppm}$ and similar to that reported in the literature ${ }^{13}$ and were not associated with any indications of toxicity (see table 3 , limit of detection $0.01 \mathrm{ppm}$ ).

Although not detected using EDX (which may not have been sufficiently sensitive to detect the low concentration of silver), acid extraction and ICP-MS suggested that $\sim 20 \%$ of the original silver loading was residual on the pacemaker titanium surfaces after 12 weeks of implantation.

\section{DISCUSSION}

This study presents an in vivo ovine model demonstrating feasibility of a silver ion-based antimicrobial surface treatment on pacemakers while maintaining pacing function at 12 weeks. The study also provides preliminary evidence of the biocompatibility of the surface treatment, recognising the small sample size used in this proof of concept study. This study showed no detectable difference between control and antimicrobially processed pacemakers with respect to the titanium and tissue reactivity in a prolonged ovine model simulating human pacing conditions using devices from four different manufacturers. The antimicrobially processed pacemakers did not affect the biocompatibility of the pacemakers used in this small study, and none of the implants became infected, despite being exposed to a prolonged in vivo model allowing natural bacterial colonisation. There was no difference in the tissue response (control and antimicrobially processed pacemakers), as judged by the appearance of the postmortem capsule and by analysing the capsule using histology. Semiquantitative scores for cellularity, vascularity and collagen production in the capsule showed no significant difference between antimicrobially processed and control pacemakers. Furthermore, qualitative measurements of the capsule thickness showed that there was no difference between antimicrobially processed and control pacemakers.

Quantitative measurements of the number of macrophages within the capsule were variable, though overall there was no difference in the capsules surrounding the antimicrobial processed and control implants. Twelve weeks appear to be a reasonable experimental time for firm tissue healing and complete and stable capsule formation with vascularisation. Although not the subject of this study, the model could be considered relevant to cases of reimplantation, where device-related infection may be of enhanced concern, as there appears to be no negative effect on biocompatibility in the capsule. The ovine model is feasible for pacing studies using the same subject for both active and control implants. Our strict surgical and pacing processes appear to be a sound structured investigative approach to measure for infection and healing processes. The only additional stage would be to implant the electrode into the heart via a suitable central vein to more closely simulate human conditions and to test for defibrillator activity of ICDs used, sensing parameters and lowest pacing thresholds. There are no non-standard techniques or additional materials required when handling the antimicrobial pacemaker during implantation. This is in contrast to the approach used by Medtronic and their TYRX antibacterial envelope, which is an additional material to handle and manipulate during implantation of the pacemaker. The silver ion-based surface treatment has the potential to provide a broader antimicrobial spectrum than the minocycline and rifampicin antibiotic combination used on the antibacterial envelope.

Whereas there was no evidence of any silver remaining on the implant surface (nor within any of the capsules) using EDX, acid extraction and ICP-MS analysis estimated $\sim 20 \%$ silver remaining on the pacemaker surface with no evidence of local colonisation, that is biofilm. This is consistent with in vitro elution studies performed using this silver ion antimicrobial surface, which shows an initial burst release of silver that then reduces in exponential manner over a period of weeks to months. The elution rate is to a large extent controlled by the buffering effect of insoluble silver chloride, limiting the local concentration of silver to well below reported toxic levels.

There were a number of limitations with this study. There were no infections observed in either device group suggesting the antimicrobial pacemaker surface was no worse than control, but no statistical claim can be made with regard to antimicrobial effectiveness or biocompatibility as numbers of subjects were small in this proof of concept study. Although this was not the primary focus of study, an in vivo infection model is conceivable that adds a controlled inoculum level into the implant pocket, and the viable bacteria count is quantified at a defined time point. Such an in vivo study model has previously been performed using small inoculated disc samples and demonstrated a statistically significant reduction in viable bacteria count for the silver ion antimicrobial surface. Although the current study used four different manufacturers' pacemakers, the number of devices used was low, of simpler complexity (single chamber, basic pacemakers) and was relatively short term, so did not investigate the effects of long-term pacemaker implantation. The number of blood and tissue samples analysed for silver content were limited, and no statistical claims can be made in this proof of concept study. We did not place the lead into veins or allow sensing functions to be tested or remote unipolar mode pacing as the leads 
were not intracardiac. Pacing times were also somewhat shorter without need for initial radiology imaging, and retrieval of the device and electrodes was simpler than human settings. ${ }^{14}$

Whereas the assessment of inflammatory effects of implants on the cells at the interface was by commonly used morphological characterisation, more definitive analysis could have been made by using immunohistochemistry to identify macrophages and various types of plasma cells.

\section{CONCLUSIONS}

This study confirms the biocompatibility and feasibility of applying the same silver ion-based antimicrobial surface technology to a titanium pacemaker surface while maintaining pacing function in a robust and unique ovine model over a follow-up of 12 weeks. This promising new approach does not change standard pacemaker implant techniques, adds no procedural delays and is worthwhile researching further to tackle the growing problem of implantable cardiac electronic implantable device infection rates. Previous studies have demonstrated that this silver ion-based antimicrobial surface technology (Agluna) is proving to be safe and clinically effective in reducing the incidence of infection in limb salvage surgery. This positive clinical experience, along with the current study, provides confidence that applying this surface treatment to pacemakers in a clinical application is a feasible route forwards to take the product to market.

Funding This work was possible through funding from Accentus Medical Ltd and a grant from the Technology Strategy Board.

\section{Competing interests None declared.}

Provenance and peer review $A B$ is a scientific advisor to Accentus Medical $\mathrm{Ltd}$, is/has been an advisor to other device and pharmaceutical companies and government agencies, is an R\&D Deputy Director for Royal Free London NHS Trust and has received honoraria for advisory and educational roles and grants to conduct research from these partners. AA is a researcher for Royal Free London NHS Trust. GB is Professor of Bioengineering at the Institute of Orthopaedics and Musculo-Skeletal Science (UCL), and is a scientific advisor to Accentus Medical Ltd. JA, DL, JS and PA are employees of Accentus Medical Ltd.

Data sharing statement № additional data are available.

Open Access This is an Open Access article distributed in accordance with the Creative Commons Attribution Non Commercial (CC BY-NC 4.0) license, which permits others to distribute, remix, adapt, build upon this work non-commercially, and license their derivative works on different terms, provided the original work is properly cited and the use is non-commercial. See: http://creativecommons.org/ licenses/by-nc/4.0/

(c) Article author(s) (or their employer(s) unless otherwise stated in the text of the article) 2017. All rights reserved. No commercial use is permitted unless otherwise expressly granted.

\section{REFERENCES}

1. Sohail MR, Wilson WR, Baddour LM. Infections of non-valvular cardiovascular devices. In: Mandell GL, Bennett JE, Dolin R, eds. Mandell, Douglas, and Bennett's principles and practice of infectious diseases. Philadelphia: Churchill Livingstone/Elsevier, 2010:1127-42.

2. Sohail MR, Uslan DZ, Khan AH, et al. Management and outcome of permanent pacemaker and implantable cardioverter-defibrillator infections. J Am Coll Cardiol 2007;49:1851-9.

3. Baddour LM, Epstein AE, Erickson CC, et al. Update on cardiovascular implantable electronic device infections and their management: a scientific statement from the American Heart Association. Circulation 2010;121:458-77.

4. Dababneh AS, Sohail MR. Cardiovascular implantable electronic device infection: a stepwise approach to diagnosis and management. Cleve Clin J Med 2011;78:529-37.

5. Reynolds MR, Cohen DJ, Kugelmass AD, et al. The frequency and incremental cost of major complications among medicare beneficiaries receiving implantable cardioverter-defibrillators. J Am Coll Cardiol 2006;47:2493-7.

6. Darouiche RO. Treatment of infections associated with surgical implants. N Engl J Med 2004;350:1422-9.

7. Ferguson TB, Ferguson CL, Crites K, et al. The additional hospital costs generated in the management of complications of pacemaker and defibrillator implantations. J Thorac Cardiovasc Surg 1996;111:742-52.

8. Sohail MR, Henrikson CA, Braid-Forbes MJ, et al. Mortality and cost associated with cardiovascular implantable electronic device infections. Arch Intern Med 2011;171:1821-8.

9. Wafa H, Grimer RJ, Carter SR, et al. Will silver solve the problem of infection around high risk prostheses? Presentation to British Orthopaedic Oncology Meeting, May, 2011.

10. Wafa H, Grimer RJ, Reddy K, et al. Retrospective evaluation of the incidence of early periprosthetic infection with silver-treated endoprostheses in high-risk patients: case-control study. Bone Joint J 2015;97-B:252-7.

11. Stokes KB, Berthelson WA, Davis MW. Metal catalyzed oxidative degradation of implanted polyurethane devices. In: Gebelein CG, ed. Advances in biomedical polymers. New York: Plenum Press, 1987:159-69.

12 Ellenbogen KA, Neal Kay G, Lau C-P, et al. Clinical cardiac pacing, defibrillation, and resynchronization therapy. 4th edn. USA: Elsevier, 2011. Chapter 4.

13. Hardes J, Ahrens H, Gebert C, et al. Lack of toxicological sideeffects in silver-coated megaprostheses in humans. Biomaterials 2007;28:2869-75.

14. Zhou X, Jiang $\mathrm{H}$, Ma J, et al. Comparison of standard and modified transvenous techniques for complex pacemaker lead extractions in the context of cardiac implantable electronic device-related infections: a 10-year experience. Europace 2013;15:1629-35. 